\title{
Intercomparison of 15 aerodynamic particle size spectrometers (APS 3321): uncertainties in particle sizing and number size distribution
}

\author{
Sascha Pfeifer ${ }^{1}$, Thomas Müller $^{1}$, Kay Weinhold ${ }^{1}$, Nadezda Zikova $^{2}$, Sebastiao Martins dos Santos ${ }^{3}$, \\ Angela Marinoni ${ }^{4}$, Oliver F. Bischof ${ }^{5}$, Carsten Kykal ${ }^{5}$, Ludwig Ries ${ }^{6}$, Frank Meinhardt ${ }^{7}$, Pasi Aalto $^{8}$, \\ Nikolaos Mihalopoulos ${ }^{9,10}$, and Alfred Wiedensohler ${ }^{1}$ \\ ${ }^{1}$ Leibniz Institute for Tropospheric Research, Leipzig, Germany \\ ${ }^{2}$ Institute of Chemical Process Fundamentals, Academy of Science, Prague, Czech Republic \\ ${ }^{3}$ Joint Research Center, Ispra, Italy \\ ${ }^{4}$ Institute for Atmospheric Sciences and Climate, Bologna, Italy \\ ${ }^{5}$ TSI GmbH, Particle Instrument, Aachen, Germany \\ ${ }^{6}$ Federal Environment Agency, GAW Station Zugspitze/Hohenpeissenberg, Platform Zugspitze, Germany \\ ${ }^{7}$ Federal Environment Agency, Platform Schauinsland, Germany \\ ${ }^{8}$ Department of Physics, University of Helsinki, Helsinki, Finland \\ ${ }^{9}$ National Observatory of Athens, Athens, Greece \\ ${ }^{10}$ Department of Chemistry, University of Crete, Heraklion, Greece \\ Correspondence to: Sascha Pfeifer (pfeifer@tropos.de)
}

Received: 11 September 2015 - Published in Atmos. Meas. Tech. Discuss.: 3 November 2015

Revised: 4 March 2016 - Accepted: 15 March 2016 - Published: 7 April 2016

\begin{abstract}
Aerodynamic particle size spectrometers are a well-established method to measure number size distributions of coarse mode particles in the atmosphere. Quality assurance is essential for atmospheric observational aerosol networks to obtain comparable results with known uncertainties. In a laboratory study within the framework of ACTRIS (Aerosols, Clouds, and Trace gases Research Infrastructure Network), 15 aerodynamic particle size spectrometers (APS model 3321, TSI Inc., St. Paul, MN, USA) were compared with a focus on flow rates, particle sizing, and the unit-tounit variability of the particle number size distribution.

Flow rate deviations were relatively small (within a few percent), while the sizing accuracy was found to be within $10 \%$ compared to polystyrene latex (PSL) reference particles. The unit-to-unit variability in terms of the particle number size distribution during this study was within $10 \%$ to $20 \%$ for particles in the range of 0.9 up to $3 \mu \mathrm{m}$, which is acceptable for atmospheric measurements. For particles smaller than that, the variability increased up to $60 \%$, probably caused by differences in the counting efficiencies of individual units. Number size distribution data for particles
\end{abstract}

smaller than $0.9 \mu \mathrm{m}$ in aerodynamic diameter should only be used with caution. For particles larger than $3 \mu \mathrm{m}$, the unit-tounit variability increased as well. A possible reason is an insufficient sizing accuracy in combination with a steeply sloping particle number size distribution and the increasing uncertainty due to decreasing counting. Particularly this uncertainty of the particle number size distribution must be considered if higher moments of the size distribution such as the particle volume or mass are calculated, which require the conversion of the aerodynamic diameter measured to a volume equivalent diameter.

In order to perform a quantitative quality assurance, a traceable reference method for the particle number concentration in the size range $0.5-3 \mu \mathrm{m}$ is needed. 


\section{Introduction}

Coarse aerosol particles in the atmosphere can have a significant influence on the optical properties of the atmospheric aerosol as well as on the total particle mass concentration. Generally, aerodynamic and optical particle size spectrometers are employed in atmospheric observational aerosol networks to directly measure the number size distribution of the coarse mode particles.

The Aerodynamic Particle Sizer spectrometer (APS model 3321, TSI Inc., St. Paul, MN, USA) is based on the acceleration of aerosol particles immersed in an air flow through a nozzle (Agarwal et al., 1979; Chen et al., 1985). The time of flight (TOF) of individual particles after acceleration is determined between two laser beams. Due to their longer relaxation time, the TOF of larger particles is longer than for smaller particles. The conversion of TOF to aerodynamic particle size classes is achieved by a calibration with polystyrene latex (PSL) spheres. Compared to optical particle size spectrometers with coherent light sources, the measuring principle of an APS is not influenced by ambiguities in the relation of the detected signal to a particle size, meaning that the calibration curve has a monotonic response over its full size range. Due to the measuring principle of the APS model 3321 made by TSI, it is however possible to measure the aerodynamic (TOF) and optical properties (scattered light) of individual particles at the same time in the so-called "correlated mode".

Nevertheless, the measurements of the aerodynamic particle size spectrometer can be influenced by a variety of errors, depending on the version or type. In general, the sizing accuracy is known and has been published by Peters and Leith (2003). The issue of coincidence of older versions of the APS was solved with the production of the model 3320. However, for this model, Armendariz and Leith (2002) showed a discrepancy between the results of the summed aerodynamic mode and the correlated measuring mode, which was resolved in the latest APS model 3321. However, Peters and Leith (2003) showed that this model had a lower counting efficiency than its predecessor.

Only a few of the published performance studies deal with results of more than one device of the same type; e.g. Volckens and Peters (2005) reported on a study with three units APS model 3321. In general, a better knowledge of the unitto-unit variability is essential in terms of the particle number size distribution. In particular, this aspect becomes important for analysis and interpretation of the results from observational atmospheric aerosol networks. Wiedensohler et al. (2012) have emphasized, that due to the growing number of measurement sites, quality controls are important to achieve comparability due to well-known uncertainties of the particle number size distribution.

In the framework of ACTRIS, an intercomparison workshop for aerodynamic particle size spectrometers was carried out at the facility of the World Calibration Center for
Aerosol Physics (WCCAP). This study dealt with the comparability of 15 aerodynamic particle size spectrometers in terms of their sizing accuracy and the unit-to-unit-variability of the particle size distribution in the size range from 0.6 to $5 \mu \mathrm{m}$ aerodynamic particle diameter.

\section{Laboratory setup and experimental procedure}

The core element in the measurement setup is a cubic mixing chamber with a volume of approximately $0.5 \mathrm{~m}^{3}$. This mixing chamber has eight outlets symmetrically arranged on the bottom plate. Unfortunately, this number of outlets limits the number of devices per run. A fan in the middle of the chamber ensures a spatially well-distributed aerosol. Test measurements with condensation particle counters assured that all outlets provide equal particle number concentrations.

In this intercomparison study, 15 units APS model 3321 (TSI Inc.) have been analyzed. An overview of all devices is given in Table 1. For the majority of devices, the last official calibration from manufacturer is not older than 3 years. Because of the limited number of chamber outlets, the devices were divided into two groups using one device (TROPOS F) as a relative reference in both runs.

In both runs, eight devices were mounted vertically underneath the individual outlets (see Fig. 1). This arrangement basically ensures no particle losses due to impaction or sedimentation from the mixing chamber to the individual devices. For all devices, a special attachment for the inlet was used, which decouples the aerosol flow $\left(1 \mathrm{~L} \mathrm{~min}^{-1}\right)$ and the sheath flow $\left(4 \mathrm{~L} \mathrm{~min}^{-1}\right)$. This reduces the total aerosol flow rate from the chamber to all devices from 40 to $8 \mathrm{~L} \mathrm{~min}^{-1}$ and avoids any aspiration effects at the inlet of the aerosol flow. The data acquisition for all devices was done simultaneously and exactly synchronized by custom-written software.

For analyzing the sizing accuracy, PSL spheres have been re-suspended, using a nebulizer in combination with a silicagel aerosol diffusion dryer. To optimize the experimental design, the sampling matrix of the PSL size calibrations has been done with two mixtures of three different PSL particle sizes $(0.7,1.0$ and $2.0 \mu \mathrm{m}$ or $0.9,1.6$ and $3.0 \mu \mathrm{m}$, respectively).

To obtain the unit-to-unit variability of the aerodynamic particle number size distribution over a wide particle size range, two procedures were carried out: (a) overnight measurements of the ambient aerosol and (b) by using a custommade coarse-mode-nebulizer to produce coarse mode ammonium sulfate particles up to $5 \mu \mathrm{m}$ in aerodynamic particle diameter.

\section{Results}

The quality of an APS in terms of sizing accuracy or particle number concentration (distribution) strongly depends on its aerosol and sheath flow rates. The manufacturer specifies the 
Table 1. Overview of compared TSI 3321 devices of the specific institute (Institute of Chemical Process Fundamentals ICPF, Institute for Atmospheric Sciences and Climate ISAC, Joint Research Center JRC, Navarino Environmental Observatory NEO, Leibniz Institute for Tropospheric Research TROPOS, Umweltbundesamt UBA, University of Helsinki UHEL) and sorted/indexed by age.

\begin{tabular}{lllll}
\hline ID & Firmware & Date of construction & Last calibration & Run \\
\hline ICPF A & 1.12 13-DEC-2001 & October 2000 & May 2002 & 2 \\
ICPF B & 1.12 13-DEC-2001 & January 2001 & June 2008 & 2 \\
ISAC & 4.00 27-DEC-2004 & June 2013 & June 2013 & 2 \\
JRC A & 1.12 13-DEC-2001 & January 2002 & July 2014 & 2 \\
JRC B & 4.00 27-DEC-2004 & August 2005 & April 2014 & 2 \\
NEO & 4.00 27-DEC-2004 & August 2006 & July 2012 & 2 \\
TROPOS A & 1.12 13-DEC-2001 & October 1997 & August 2012 & 1 \\
TROPOS B & 1.12 13-DEC-2001 & October 2001 & September 2011 & 1 \\
TROPOS C & 4.00 27-DEC-2004 & November 2007 & January 2013 & 1 \\
TROPOS D & 4.00 27-DEC-2004 & September 2008 & May 2014 & 1 \\
TROPOS E & 4.00 27-DEC-2004 & December 2011 & arch 2012 & 1 \\
TROPOS F & 4.00 27-DEC-2004 & May 2014 & May 2014 & 1 \& 2 \\
UBA A & 4.00 27-DEC-2004 & December 2011 & December 2011 & 1 \\
UBA B & $4.0027-D E C-2004$ & December 2011 & December 2011 & 1 \\
UHEL & 1.12 13-DEC-2001 & May 2001 & June 2005 & 2 \\
\hline
\end{tabular}

aerosol flow with $1.0 \pm 0.1 \mathrm{~L} \mathrm{~min}^{-1}$ and the sheath flow with $4.0 \pm 0.1 \mathrm{~L} \mathrm{~min}^{-1}$ (TSI, 2004), although the aerosol flow is set to a tighter range of $0.995-1.005 \mathrm{~L} \mathrm{~min}^{-1}$ when units are calibrated by TSI. The as-found flow rates measured in the initial state for all devices are shown in Fig. 2. It should be noted that all devices came to the workshop as they were last used and that there was no assessment of when the last routine maintenance was performed. Only a few devices showed a significant deviation from the specified range, namely ICPF $\mathrm{A}$ and $\mathrm{B}, \mathrm{TROPOS} \mathrm{B}, \mathrm{E}$, and $\mathrm{F}$. At the end of the first round, the pump for the total flow of TROPOS $\mathrm{E}$ was found to be broken. The previous measurements for sizing accuracy or particle number size distribution were not influenced by this incident. For some devices, (ICPF A and B, JRC A and B, ISAC) the flow rates were re-adjusted to the reference values. The flow rates of TROPOS F have been left untouched, because these were the original manufacturer's settings of a unit that was just few months old at the time. No re-calibrations of the TOFs were performed. In the following sections, only the results after the flow re-adjustments are analyzed.

\subsection{Sizing accuracy}

The mean particle diameters were determined by fitting a multi-modal logarithmic function to the measured particle number distributions of the re-suspended PSL mixtures. These results were compared to aerodynamic diameters calculated from the manufacturer's data, considering the Cunningham slip correction, but no ultra-Stokes effects (Wang and John, 1987). The relative deviation between both values is shown in Fig. 3.

For the majority of devices, the deviations in terms of sizing are less than $10 \%$, with a few exceptions. ICPF A shows significantly higher values over a wide range. This may be

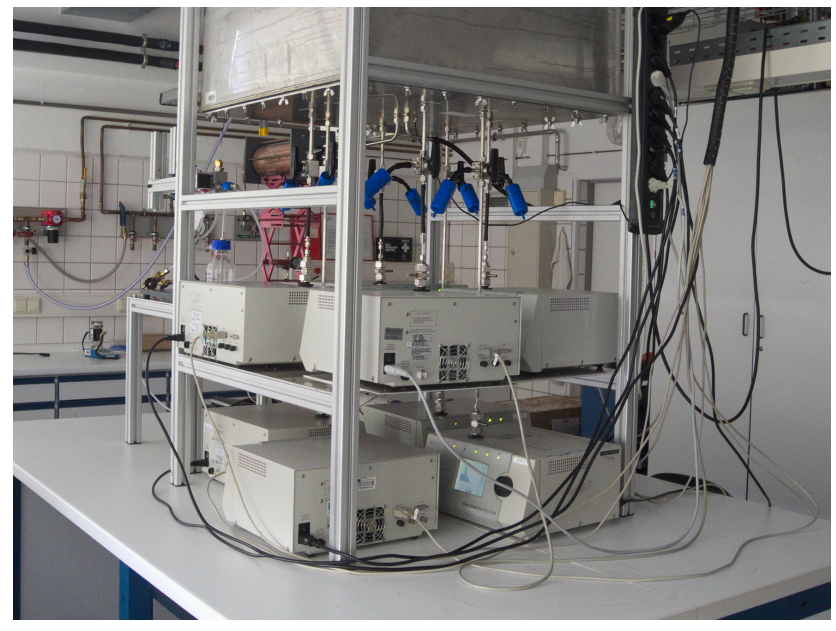

Figure 1. Photo of the measuring setup for the intercomparison of eight units APS 3321.

a result of its flow re-adjustment, while the TOF calibration was untouched. Also for NEO, the internal TOF calibration parameters seem unsuitable and incorrect for the readjusted flow rates. On average, an optimum for $1.6 \mu \mathrm{m}$ is noticeable. For smaller particles, unsystematic deviations are visible, whereas for larger particles the results seems to be systematic too low and some more outliers are noticeable. For particles smaller than $0.8 \mu \mathrm{m}$, the sizing accuracy can be distorted by the counting efficiency of the device, which was previously shown for the older 3310 model APS (Karg et al., 1991). The results for larger PSL spheres might be influenced by poor counting statistics with relative deviations up to $5 \%$ for $3 \mu \mathrm{m}$, based on the decreasing concentration. More importantly, the number of resolved bins of the measured par- 


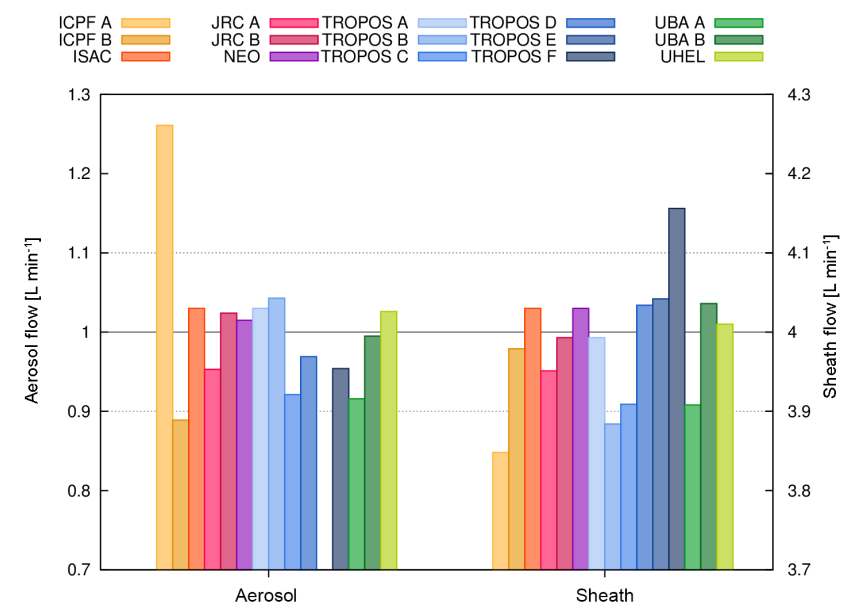

Figure 2. Measured aerosol and sheath flow rates of the initial state.

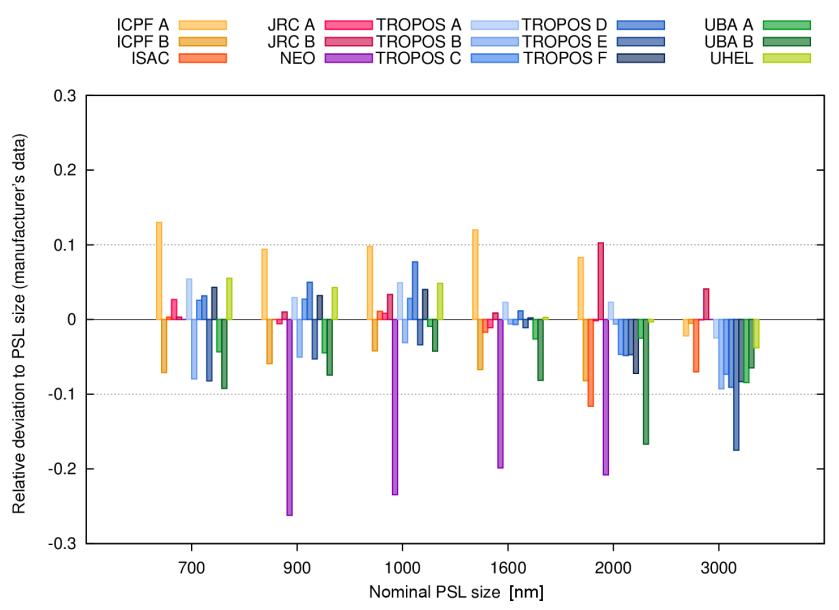

Figure 3. Relative deviation of the measured aerodynamic diameter of six PSL sphere sizes.

ticle number size distribution for larger PSL spheres is reduced compared to smaller particles. This aspect makes the non-linear fitting of log-normal distributed particle number size distribution fault-prone and reduces the statistical significance of the resulting parameters. In general, the results also depend on the applied conversion formula to calculate the aerodynamic diameters, especially considering the ultraStokes effects for larger particles (Wang and John, 1987).

\subsection{Comparability of particle number size distributions}

The sizing first had to be corrected to merge the results of the runs of the different sets of instruments and to make them comparable. This was done to decouple the variability in sizing from the concentration measurements. Because of the diverse influences for smaller and larger particles, the sizing for the entire particle size range was corrected using only the results from $1.6 \mu \mathrm{m}$ PSL sizing check. After this correction, the particle number size distributions of the devices from the first run were corrected binwise multiplicative to the second run using TROPOS F as a relative reference instrument. Based on Poisson counting statistics, for further analysis the range up to $5 \mu \mathrm{m}$ is acceptable with a relative error smaller than $1 \%$. To analyze the variability for the whole particle size range, a mean particle number size distribution was calculated. For each size bin, a percentile filter was used (rejecting the first and the last three data points) to reduce the influence of outliers. The average and standard deviation were calculated with the nine remaining values.

The results are shown in Fig. 4. The particle number size distributions for the 15 devices strongly deviate, especially in the sub-micron size range. For the lowest size channels, the deviation is up to a factor of 10 . The mean relative deviation (95\% confidence interval) decreases steadily from approximately $60 \%$ for the smallest size channels and reaches a minimum with values of $10-20 \%$ in the size range from 0.9 up to $3 \mu \mathrm{m}$. For larger size channels the mean relative deviation increases up to $40 \%$ for ambient aerosol and $130 \%$ for ammonium sulfate, respectively. These uncertainties should not be overvalued, because of the strongly decreasing particle number concentration causing poor counting statistics in this size range. Any insufficient correction for sizing could be misinterpreted as an error of concentration. This aspect is particularly true for ICPF B and JRC B for ammonium sulfate and ICPF A and TROPOS F for ambient aerosol. This supposed deviation in sizing could not be validated by PSL, because of the upper limit of $3 \mu \mathrm{m}$ defined in this study.

Four devices stood out from the general behaviour and showed a poorer performance, especially in the size range with the smallest variability among the units overall (0.9$3.0 \mu \mathrm{m})$. NEO deviated for particles smaller than $1.6 \mu \mathrm{m}$ for both samples, somewhat more significant for ambient aerosol. UBA A deviates even for particles smaller than $2 \mu \mathrm{m}$, especially for ammonium sulfate up to $-60 \%$. For ammonium sulfate, UBA B shows the same behaviour like UBA A but over a smaller size range. In contrast, the concentration for ambient aerosol in the size range around $1 \mu \mathrm{m}$ is somewhat high. Over a wide range, the number size distribution of TROPOS D has been higher compared to other instruments. For ambient aerosol this behaviour is much more pronounced than for ammonium sulfate. Assuming technical problems and an unknown maintenance status, we excluded these devices from the further discussions.

\section{Discussion}

The relatively large unit-to-unit variability up to $60 \%$ between the particle number size distributions did not meet the expectations. The measured flow rates lay within the specified range or were re-adjusted to the reference values. On average, the size accuracy was within $10 \%$. Furthermore, although no TOF-recalibration has been performed, the devi- 

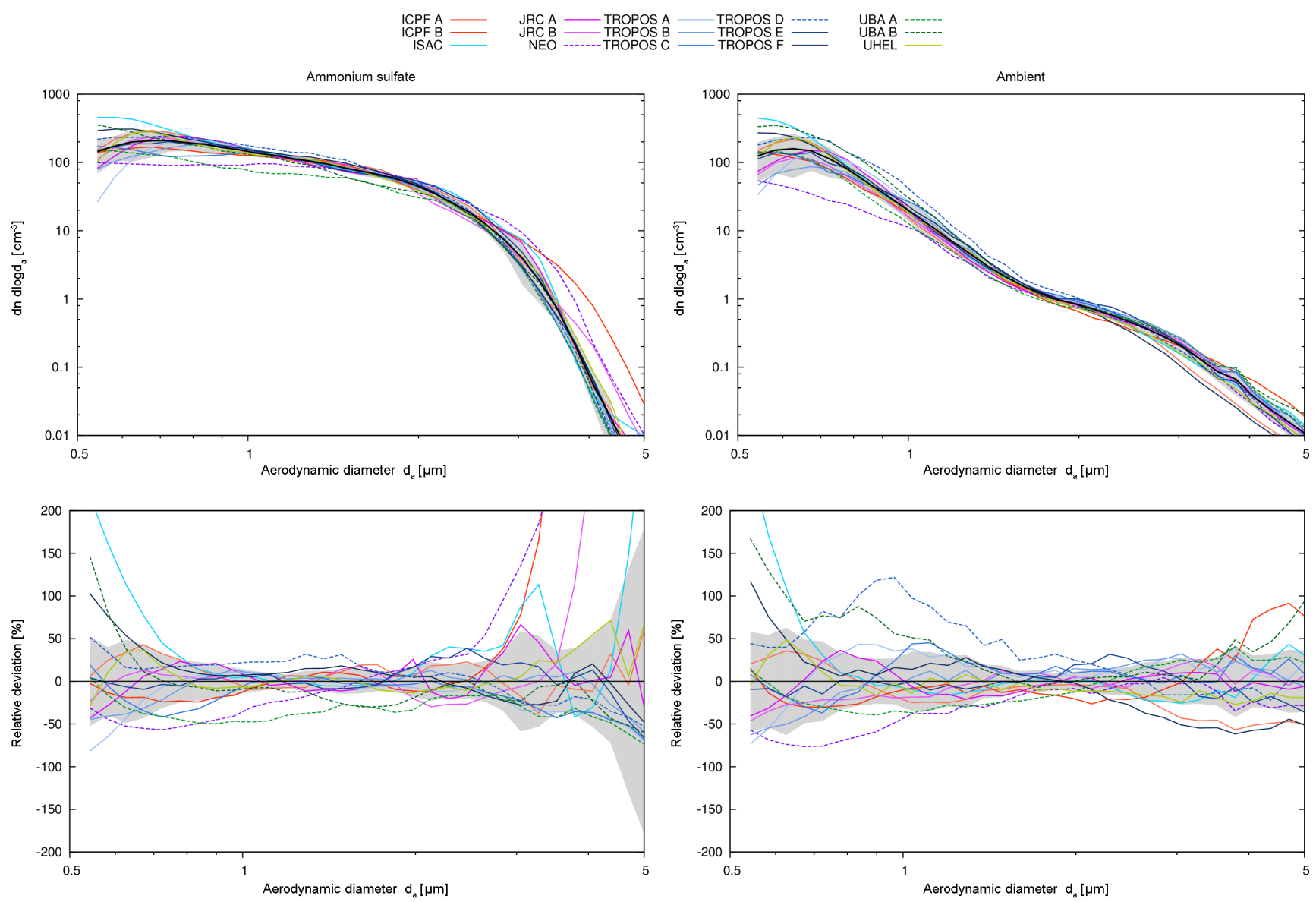

Figure 4. Merged results of both runs for ammonium sulfate (left column) and ambient aerosol (right column), particle number size distribution (upper row) and relative deviation from average (lower row). The grey shaded range is the mean deviation (95\% confidence interval) of the selected values.

ations in sizing were corrected roughly in a post-processing step.

Taking into account the specified range for the aerosol flow rate, a variation of $10 \%$ for the concentration seems to be acceptable. Because of insufficient sizing accuracy in combination with a moderately sloping particle number size distribution, the expected variability is slightly larger (approximately $20 \%$ ). With increasing slope this aspect becomes more important, e.g. for ammonium sulfate larger than $3 \mu \mathrm{m}$.

The large unit-to-unit variability of the number size distribution in the sub-micron range certainly results from individual differences in unit counting efficiencies. This issue was analyzed in several studies for previous TSI APS models as well as for the latest model 3321 (Karg et al., 1991; Armendariz and Leith, 2002; Peters and Leith, 2003; Volckens and Peters, 2005). In general, the counting efficiency of a TSI APS model 3321 is influenced by aspiration losses, transmission losses and detector errors (Volcken and Peters, 2005). The detector error is associated with low pulse height of the optical signals used for the TOF measurement. The effect can be divided into two types.
1. Just one of the two signals is lower than a certain threshold. Such events are rejected for the particle number size distribution. However, it is marked and counted by the device as "Event Type I".

2. Neither of the two signals reaches the threshold, because the particle misses the laser beam or scatters just too little light for other reasons. Such particles are completely undetected by the device.

The unit-to-unit-variability in the sub-micron range should be primarily based on these two types of detector errors. Either the general quality of the optics (cleanliness of the optical components, detector sensitivity, laser beam focusing, etc.) or the precision of the alignment of the aerosol flow and the laser beam could be a reason for this variability.

Karg et al. (1991) already showed that the counting efficiency may depend also on the sample. This aspect is reasonable in the context that the counting efficiency in the submicron range is based on the detector error. Therefore, the counting efficiency is also a function of the optical properties (primarily the complex refractive index) of the sample, which 

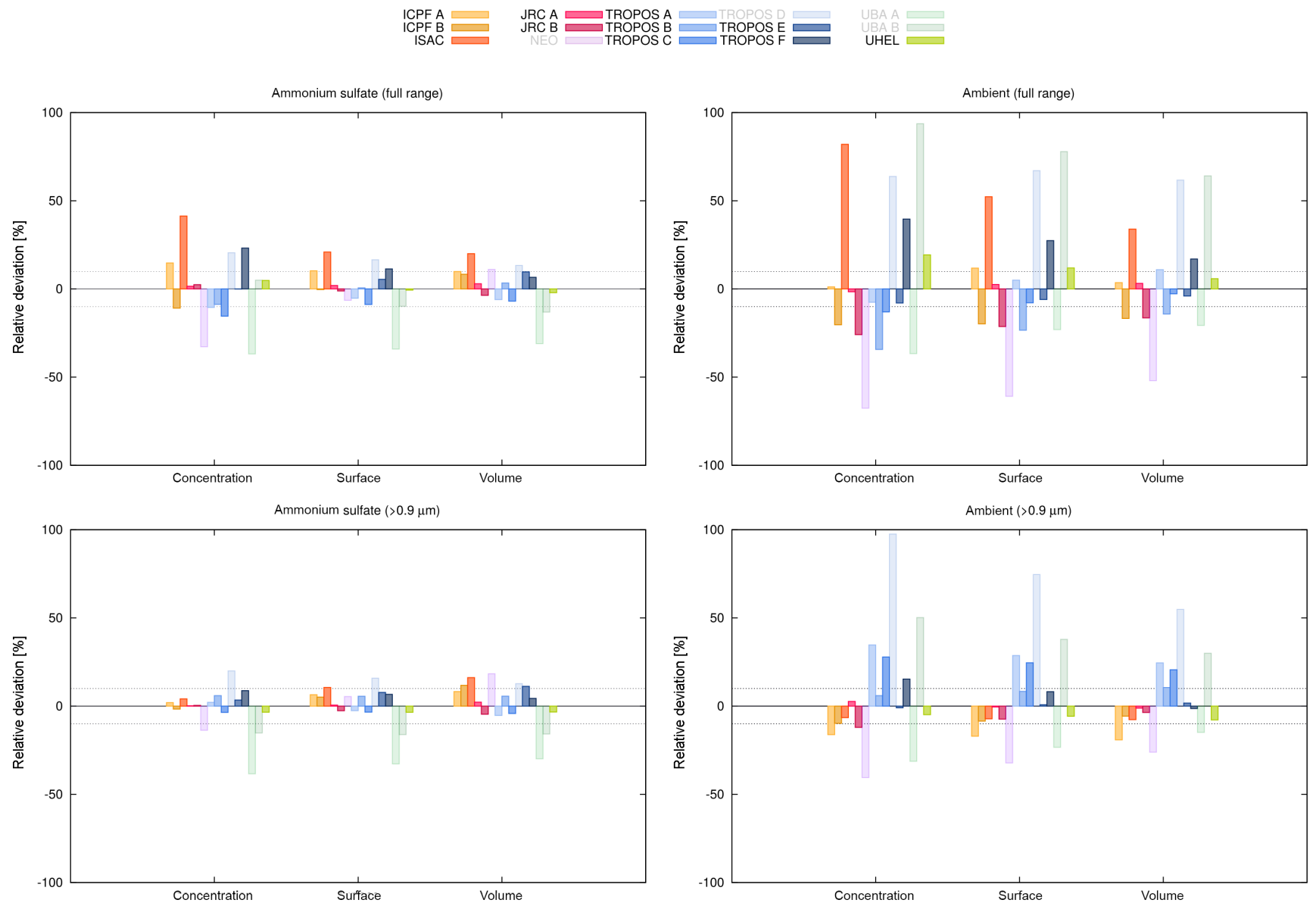

Figure 5. Relative deviation of the calculated total number, surface and volume concentration for the measured distribution relative to the averaged distribution: ammonium sulfate (left column) and ambient aerosol (right column), full size range (upper row) and for particles larger $0.9 \mu \mathrm{m}$ (lower row).

means it is rather a function of the optical diameter than the aerodynamic diameter. Extended analysis of pulse pair type or the scattering signal in the correlated mode are necessary. Although a slight deviation between the results of the two samples is noticeable, an independent measuring principle is necessary to investigate this effect. The significant deviation between laboratory generated ammonium sulfate and ambient aerosol of some devices in the coarse mode size range cannot be explained by this argumentation.

During the intercomparison, no traceable reference method for a particle number concentration was available for aerosol particles between 0.5 and $3 \mu \mathrm{m}$. Compared to other studies, e.g. Karg et al. (1991), Peters and Leith (2003), Volcken and Peters (2005), it was not possible to determine quantitatively the individual counting efficiencies. The particle number size distribution could be only qualitatively compared to each other.

The resulting deviation for the calculated integral values of total number, surface and volume concentration is shown in Fig. 5. The mean variability of ammonium sulfate is much smaller than for ambient aerosol, due to the higher concentration in the super-micron range. This is also the reason for the lower variability of the total particle number concentration compared to the total particle volume, due to the stronger weighting of larger particles. Compared to the whole particle size range, the variability for particles larger than $0.9 \mu \mathrm{m}$ is acceptable in the range of $\pm 10 \%$ for the majority of devices. For this size range, there is no significant difference between the integrating values. Only for ambient aerosol, ICPF A shows significantly lower values of $-17 \%$ and TROPOS A and $\mathrm{C}$ higher values of 29 and $24 \%$ on average. Without any further individual correction based on the counting efficiency, the particle number size distribution from the APS' lower detection limit of 0.5 up to $0.9 \mu \mathrm{m}$ should be generally considered with caution.

\section{Conclusions}

Quality controls are essential to get comparable and accurate results for atmospheric measurement networks. In the frame- 
work of ACTRIS, 15 aerodynamic particle size spectrometers were intercompared with a focus on the basic parameters: flow rates, size accuracy, and concentration.

For the majority of devices, the measured flow rates were in the specified tolerance range of $0.9-1.1$ and 3.9$4.1 \mathrm{~L} \mathrm{~min}^{-1}$, respectively. The sizing accuracy was determined using PSL spheres with six sizes in the range from 0.7 to $3.0 \mu \mathrm{m}$. With respect to the nominal PSL diameters, the mean deviation for the size accuracy was generally $\pm 10 \%$ with a systematic trend toward a negative deviation ( 0 to $-10 \%$ ) for larger particles.

The most significant differences and variability can be found for the concentration measurements. The size range up to $0.9 \mu \mathrm{m}$ is characterized by a large unit-to-unit-variability up to $60 \%$. This variability is most likely a result of individual counting efficiencies based on detector sensitivity. For the size range of 0.9 up to $3 \mu \mathrm{m}$, the variability is in the range of $10-20 \%$. This range is acceptable considering the specified range for aerosol flow rates as well as insufficient size accuracy in combination with a sloping particle number size distribution. The second issue might be a reason for the increasing variability for particles larger than $3 \mu \mathrm{m}$. A validation for the size accuracy of particles larger than $3 \mu \mathrm{m}$ was not possible in this study.

Naturally, the significant unit-to-unit variability propagates for the derived integrated values (total number, surface and volume concentration). Only for the size range larger than $0.9 \mu \mathrm{m}$, the variability is within the range of $10 \%$ for the majority of devices. Thus, without further device-specific calibration and testing the size range below $0.9 \mu \mathrm{m}$ should be rejected. Unfortunately, it was not possible to determine quantitatively the individual counting efficiencies, because no traceable reference method was available for accurate number concentrations of around $1 \mu \mathrm{m}$.

Some devices have shown an extraordinarily poor quality based on technical defects or insufficient calibration. These instruments have not been considered in the final analysis. In conclusion, a few points should be emphasized for the future, considering long-term measurements.

- Quality checks for flow rates and size accuracy should be a standard procedure in the field. After a readjustment of the flow rates, a TOF re-calibration might be needed.

- Measured particle number size distributions are influenced by counting efficiency effects. Individual correction functions are needed as a standard data processing step to get comparable results.

- For quality controls of concentration measurements and to derive such counting efficiency functions a traceable reference method is needed for number concentrations in the particle size range from 0.5 to $3 \mu \mathrm{m}$.
Acknowledgements. This work was accomplished by the European research infrastructure project ACTRIS (Aerosols, Clouds, and Trace gases Research InfraStructure Network). The research leading to these results has received funding from the European Union Seventh Framework Programme (FP7/2007-2013) project No. 262254.

We would like to thank TSI for their support during the workshop. This laboratory study was independently performed and was not co-funded by TSI.

Edited by: S. Malinowski

\section{References}

Agarwal, J. K. and Fingerson, L. M.: Real-time aerodynamic particle size measurement with a laser velocimeter, TSI Quarterly, V(1), 1979.

Armendariz, A. J. and Leith, D.: Concentration measurement and counting efficiency for the aerodynamic particle sizer 3320, J. Aerosol Sci., 33, 133-148, 2002.

Chen, B. T., Cheng, Y. S., and Yeh, H. C.: Performance of a TSI aerodynamic particle sizer, Aerosol Sci. Tech., 4, 89-97, 1985.

Karg, E., Dua, S. K., and Tschiersch, J.: Experimental counting efficiency of the TSI aerodynamic particle sizer in the submicron range, J. Aerosol Sci., 22, 351-354, 1991.

Peters, T. M. and Leith, D.: Concentration measurement and counting efficiency of the aerodynamic particle sizer 3321, J. Aerosol Sci., 34, 627-634, 2003.

TSI: Model 3321 Aerodynamic Particle Sizer Spectrometer - Instruction Manual, Revision E, TSI Inc., Shoreview, MN, USA, 2004.

Volckens, J. and Peters, P. M.: Counting and particle transmission efficiency of the aerodynamic particle sizer, J. Aerosol Sci., 36, 1400-1408, 2005.

Wang, H.-C. and John, W.: Particle density correction for the aerodynamic particle sizer, Aerosol Sci. Tech., 6, 191-198, 1987.

Wiedensohler, A., Birmili, W., Nowak, A., Sonntag, A., Weinhold, K., Merkel, M., Wehner, B., Tuch, T., Pfeifer, S., Fiebig, M., Fjäraa, A. M., Asmi, E., Sellegri, K., Depuy, R., Venzac, H., Villani, P., Laj, P., Aalto, P., Ogren, J. A., Swietlicki, E., Williams, P., Roldin, P., Quincey, P., Hüglin, C., Fierz-Schmidhauser, R., Gysel, M., Weingartner, E., Riccobono, F., Santos, S., Grüning, C., Faloon, K., Beddows, D., Harrison, R., Monahan, C., Jennings, S. G., O’Dowd, C. D., Marinoni, A., Horn, H.-G., Keck, L., Jiang, J., Scheckman, J., McMurry, P. H., Deng, Z., Zhao, C. S., Moerman, M., Henzing, B., de Leeuw, G., Löschau, G., and Bastian, S.: Mobility particle size spectrometers: harmonization of technical standards and data structure to facilitate high quality long-term observations of atmospheric particle number size distributions, Atmos. Meas. Tech., 5, 657-685, doi:10.5194/amt5-657-2012, 2012. 